\title{
Research Paper \\ Effect of 12 Weeks of Core Stability Exercises on Functional Movement and Bal- ance of Women Working in Hospitals
}

\author{
"Maryam Golmoradi Marani' ${ }^{1}$, Mahdi Khalegi², Aezam Ahmadi³, Seyyedeh Fatemeh Alavi" ${ }^{4}$
}

\author{
1. Department of Corrective Exercise and Sport Injury, Faculty of Physical Education and Sport Sciences, Kharazmi University, Tehran, Iran. \\ 2. Department of Biomechanic and Sport Injury, Faculty of Physical Education and Sport Sciences, Kharazmi University, Tehran, Iran. \\ 3. Department of Sport Physiology, Faculty of Physical Education and Sport Sciences, Kharazmi University, Tehran, Iran. \\ 4. Educational Assistant Center for Research on Occupational Diseases, Tehran University of Medical Sciences, Tehran, Iran.
}

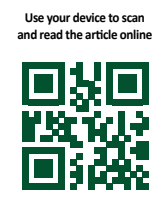

Citration: Golmoradi Marani M, Khalegi M, Ahmadi A, Alavi SF. [Effect of 12 Weeks of Core Stability Exercises on Functional Movement and Balance of Women Working in Hospitals (Persian)]. Journal of Sport Biomechanics. 2021; 7(1):122-135. https://doi.org/10.32598/biomechanics.7.2.4

https://doi.org/10.32598/biomechanics.7.2.4

Keywords:

Core stability, Dynam-

ic balance, Functional movement

\section{A B STRACT}

Objective This study aims to assess the effect of 12 weeks of core stability exercises on functional movement and balance of women working in hospitals.

Methods Participants were 34 women aged 30-50 years working in Imam Khomeini Hospital in Tehran, Iran. They were randomly divided into two groups of exercise and control. The exercise group performed core stability exercises for 12 weeks. The functional movement was evaluated using the valid Function Movement Screen (FMS) method. The dynamic balance, power, agility, speed and flexibility of subjects were also measured using $Y$ balance test, Sargent Jump Test, agility T-test, 20-meter running test, and seated bending forward test, respectively before and after exercise. Paired T-test was used to examine the difference between pre- and post-test scores of each group. Analysis of Covariance (ANCOVA) was used to determine the effect of intervention on the study variables.

Results Results of ANCOVA showed that 12 weeks of core stability exercises significantly improved functional movement performance, dynamic balance, power, agility, speed and flexibility in women.

Conclusion Core stability exercises can improve the performance of neuromuscular system leading to proper movement of spinal, pelvic and hip joints, proper muscle balance, enhanced proximal stability, functional strength and resulting in decreased injury.

\section{$\mathbf{T}$}

Extended Abstract

\section{Introduction}

he health sector is the largest employment sector in most countries, with the majority of the workforce being women. One of the characteristics of working in a hospital is that all tasks are performed manually, which causes early onset of musculo- skeletal disorders among its staff. Today, the prevention of musculoskeletal disorders has become a national necessity and priority in most countries [1].

Many studies have shown the importance of core muscle stability in human movements [2]. Core muscle strength and endurance seem to be one of the important prerequisites for most daily activities [3]. It is also believed that the weak core area causes changes in energy transfer and, as a

\section{* Corresponding Author:}

Maryam Golmoradi Marani

Address: Department of Corrective Exercise and Sport Injury, Faculty of Physical Education and Sport Sciences, Kharazmi University, Tehran, Iran.

Tel: +98 (914) 9529241

E-mail: maryam.golmoradi@yahoo.com 
result, reduces performance and increases the risk of injury to weak muscle groups [4]. Recently, core area exercises have been used by trainers as an intervention to prevent and treat injuries, as well as to correct the posture and spine [5].

The ability of individuals to maintain balance is essential for almost all daily activities [6]. Movement assessment allows health professionals to observe motor defects, asymmetry, and muscle balance [7]. Limb asymmetry increases the risk of injury; hence, it seems that before participation in any training programs there is a need for performance assessment [8]. Functional Movement Screen (FMS) method is a way to initially evaluate motor performance and help identify asymmetry, muscle imbalance, and other injury risk factors, and can easily measure the state of neuromuscular coordination $[9,10,11]$. FMS is a fast, non-invasive and inexpensive test that is easy to use and emphasizes the quality of the movement pattern $[1,12]$. Although the FMS test is more commonly used to screen athletes, it may also be used on non-athletes to assess their weaknesses in movement. The present study aims to evaluate the effect of core stability exercises on functional performance and balance of female staff in hospitals.

\section{Methods}

The study population included all female employees of Imam Khomeini Hospital aged 30-50 years. Based on the inclusion criteria, 60 were selected using a convenience sampling method and randomly divided into two groups of exercise and control. For 12 weeks, the exercise group entered performed core stability exercises while the control group engaged in their daily activities. Subjects underwent FMS, performance screening, and balance tests before and after the exercise period. For this purpose, first the height and weight of the subjects were measured in centimeters and kilograms. Then, Y-balance test for dynamic balance,
Agility T-Test for agility, seated forward bend for flexibility, Vertical Jump Test for body power, 20-m running test for Speed, and FMS for movement performance were carried out. Good Intra-rater and inter-rater reliability have been reported for FMS [13]. The total score of FMS is 21; a score less than 14 indicates that a person is prone to injury [14]. The core stability exercise protocol was according to Jeffreys [15]. After the end of exercise, the above mentioned tests were carried out again. It should be noted that the number of samples studied was reduced from 60 to 34 due to lack of cooperation. To analyze the collected data, first the paired test was used to show whether the post-test scores of each group were different from their pre-test scores. Then, in order to investigate the between-group difference, the effect of the independent variable (core stability exercise) on the dependent variables was examined using the analysis of covariance (ANCOVA) to determine whether the intervention, without interacting with the confounding factor (pretest score), could have a significant effect on the dependent variables or not.

\section{Results}

The results of paired test showed that after 12 weeks of core stability exercise, there was a significant difference in the scores of test between the pre-test and post-test phases in the exercise group, such that the scores earned in the posttest phase were significantly higher than the pre-test scores for all variables $(\mathrm{P}<0.05)$. In the control group, there was no significant difference $(\mathrm{P}>0.05)$. In order to investigate the effect of central stability exercises on dependent variables, ANCOVA was used to for assessing between-group differences. It requires that the assumptions of homogeneity of variances, linearity, and homogeneity of regression slopes be observed in the study variables. The results showed that the assumption of homogeneity of variances, homogeneity of regression slopes $(\mathrm{P}>0.05)$, as well as the assumption

Table 1. Results of ANCOVA to evaluate the effectiveness of core stability exercises on the dependent variables

\begin{tabular}{|cccccc|}
\hline Group & Sum of Squares & Df & F & Sig. & Observed Power \\
\hline Functional movement & 309.140 & 1 & 66.78 & 0.001 & 0.79 \\
\hline Balance & 699.123 & 1 & 14.42 & 0.001 & 0.91 \\
\hline Speed & 75.19 & 1 & 51.77 & 0.001 & 0.93 \\
\hline Power & 889.71 & 1 & 36.84 & 0.001 & 0.84 \\
\hline Agility & 795.27 & 1 & 19.18 & 0.001 & 0.67 \\
\hline Flexibility & 50.122 & 1 & 38.45 & 0.02 & 0.84 \\
\hline
\end{tabular}


of linearity of the correlation between the covariate and the independent variable $(\mathrm{P}<0.05)$ were confirmed in all study variables. The results of ANCOVA related to posttest scores showed that the significance level was less than 0.05 ; hence, the mean difference between the groups in the post-test phase was significant after controlling the possible effect of the pre-test score (Table 1). Therefore, as can be seen, core stability exercises had a significant effect on all study variables $(\mathrm{P}<0.05)$.

\section{Discussion and Conclusion}

The purpose of the present study was to investigate the effect of a core stability exercise program on functional performance and balance in women aged 30-50 years working in a Hospital. The results revealed that 12 weeks of core stability exercise improved their functional performance, dynamic balance and other fitness factors such as speed, power, agility and flexibility in women. Regarding the effect of core stability exercises on the improvement of functional performance, our findings are consistent with the findings of many previous studies. Goss et al. showed that during a six-week functional training program, a significant increase in FMS score was observed [16]. Kiesel et al. also concluded that a period of training program increased FMS scores in $51.6 \%$ of football players [17]. Shirey et al. stated that voluntary activation of the core muscles had positive effects in reducing the risk of limb injuries [18].

Because balance in the motor chain relies on movement and feedback strategies, facilitating simultaneous contraction of the muscles around the lumbar vertebrae, such as oblique muscle, transverse abdominis, and erector spinae muscles, may increase the stability of vertebrae $[19,20]$. The results of the present study are consistent with the results of Johnson et al. and Carpes et al. regarding the significant effect of trunk muscle strengthening exercises on improving dynamic balance $[21,22]$ as well as the findings of Imai et al., Carr and Shepherd, Samson, and Abdi and Sadeghi who reported the effect of core stability training program on dynamic balance [23-26].

It can be concluded that the core stability exercise program used in this study is a proper method with no complications and can improve the efficiency of the neuromuscular system. This causes optimal movement of the spinal, pelvic, and hip joints along the motor-function chain; proper muscle balance, enhanced proximal stability and functional strength, and ultimately reduces the risk of injury.

\section{Ethical Considerations}

\section{Compliance with ethical guidelines}

All ethical principles are considered in this article. The participants were informed about the purpose of the research and its implementation stages. They were also assured about the confidentiality of their information. They were free to leave the study whenever they wished, and if desired, the research results would be available to them.

\section{Funding}

This study was extracted from a research project approved by Tehran University of Medical Sciences.

Authors' contributions

All authors equally contributed to preparing this article.

\section{Conflicts of interest}

The authors declared no conflict of interest.

\section{Acknowledgements}

The authors would like to thank the participants and the personnel of Imam Khomeini Hospital in Tehran for their cooperation 
مقاله يخووهشى

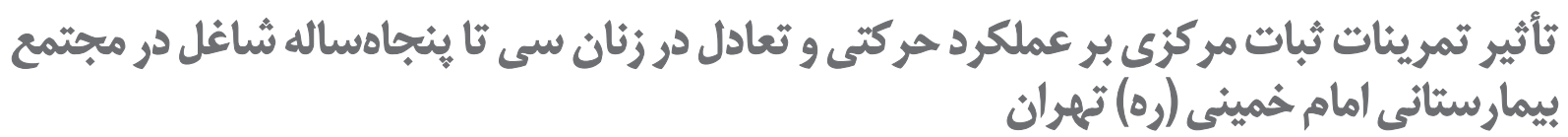

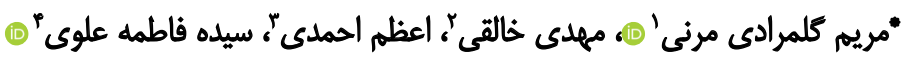
1. كروه آسيبشناسى و حركات اصلاحى، دانشكده تربيثبدنى و علوم ورزشى، دانشَاه خوارزمى، تهران، يراني.

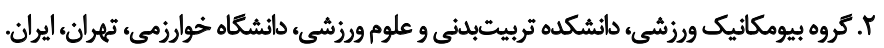

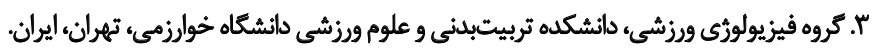

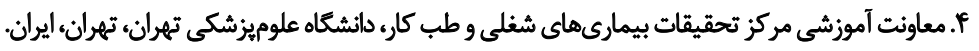

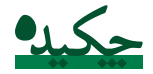

هدف هدف از تحقيق حاضر، بررسى تأثير تمريثات ثبات مركزى بر عملكرد حركتى و تعادل كاركنان زن سى تا بنجاه ساله مجتمع بيمارستانى امام خمينى (ره) تهران بود.

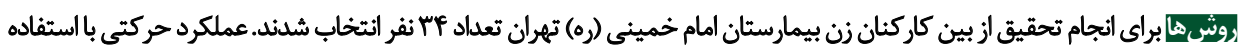

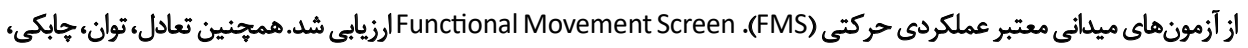

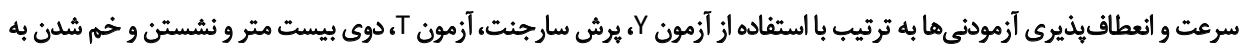

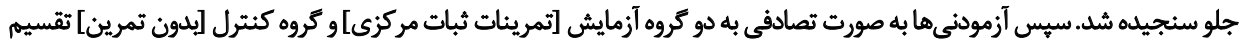

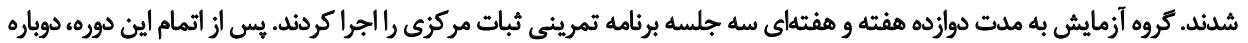

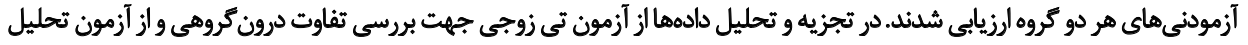

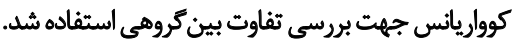

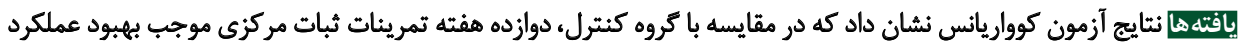

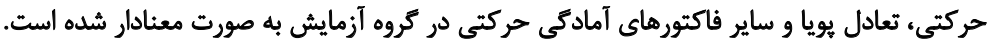

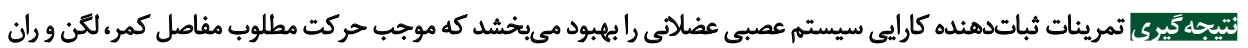

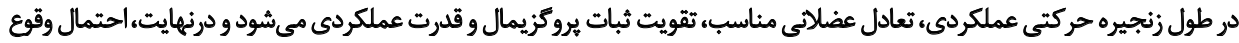

و ازجمله مهمترين مسائلى است كه اركونوميستها در سراسر

مقلمه

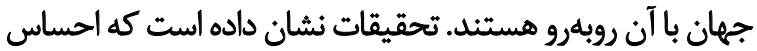

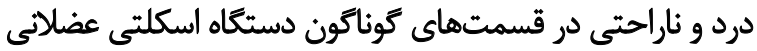

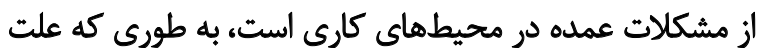
بخش بهداشت و درمان، بزركترين بخش از لحاظ استخدام

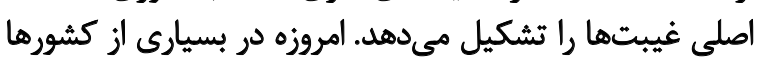

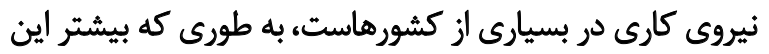

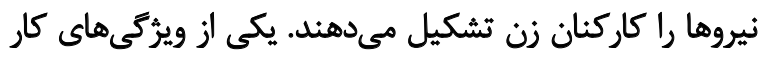

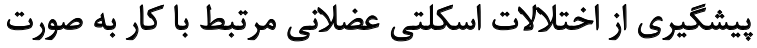

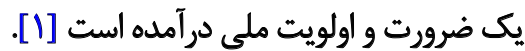

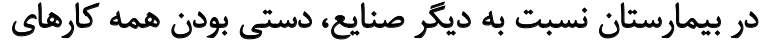

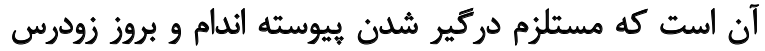

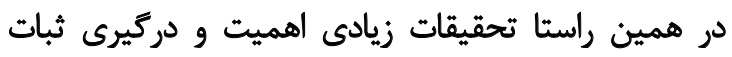

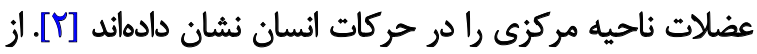

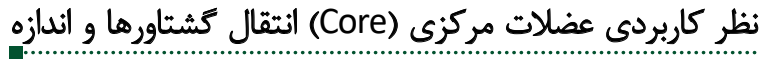

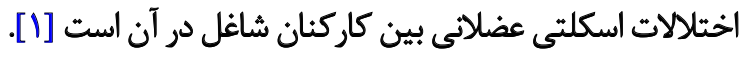

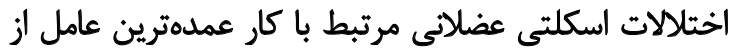

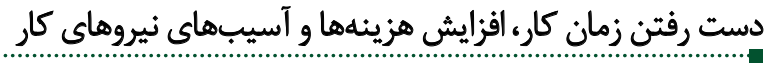

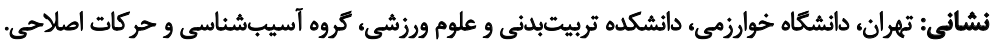


جسمانى امكان مشاهده نقصهاي حركتى، عدم تقارن و تعادل

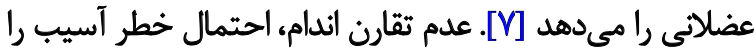

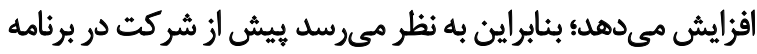

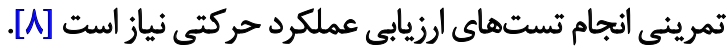
آزمونهاى غربالكرى عملكرد حركتى، راهى براى ارزيابى

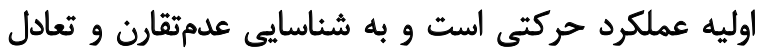

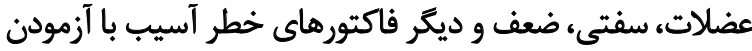
حركتيذيرى و استحكام (ثبات) لكن، شائه، زانو، كمر و مئجيا

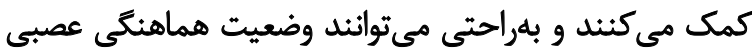

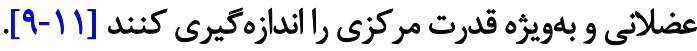
آزمون غربالكرى عملكرد حركتى، آزمونى سريع، غيرتهاجمى آجي

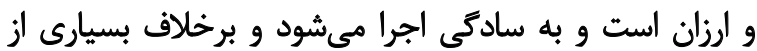

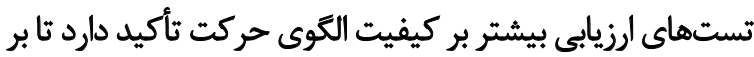

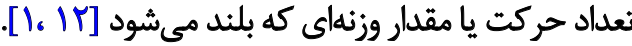

تيهن و همكاران، يايايي درون آزمونكر و بين آزمونكر متوسط تا

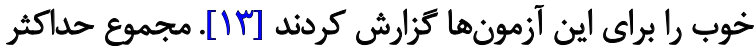

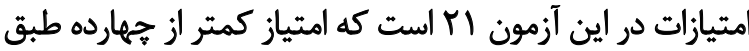

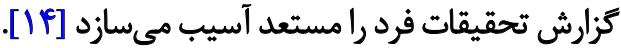
با وجود اينكه تست آزمون غربالكرى عملكرد حركتى بيشتر

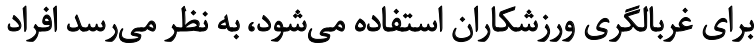

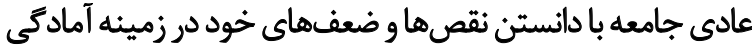

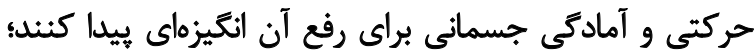

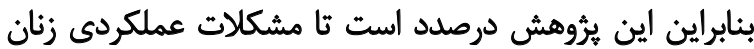

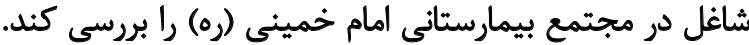

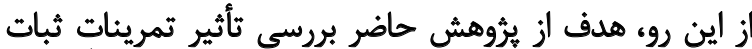

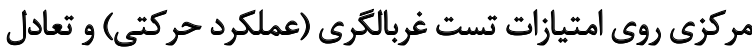

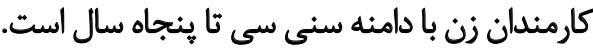

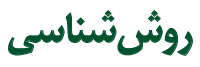

جامعه آمارى اين يروهش شامل تمام كارمندان زن بيمارستان

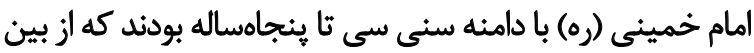

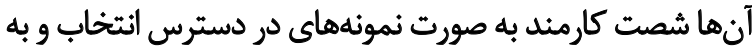

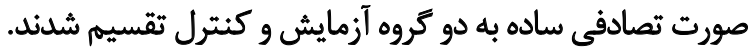

هنكام اجراى طرح ع نفر از نمونهها به دلايل شخصى مايل

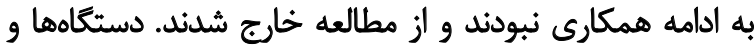

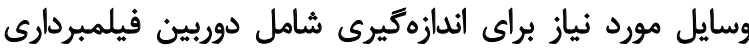

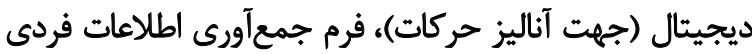

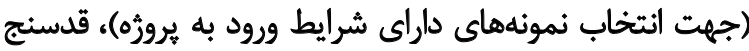

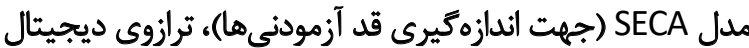

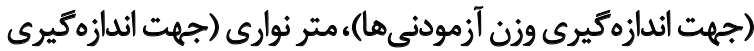

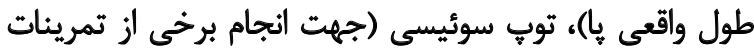

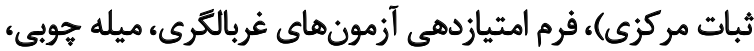

حركت زاويهاي را بين اندامهاى بايينتثنه و بالاتنه تسهيل مى كند

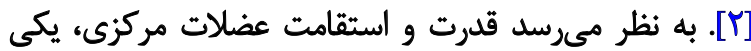

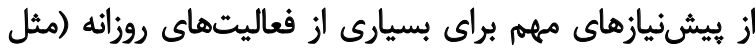

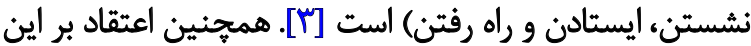

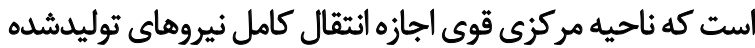

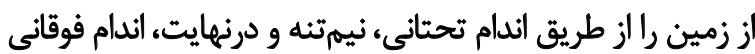

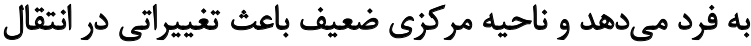

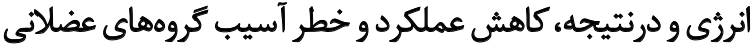

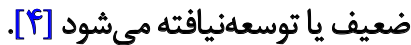
در سال هاى اخير ناحيه هركزى بدن و تمرينات مربوط به به تقويت

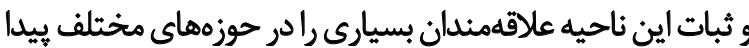

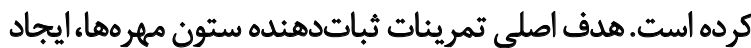

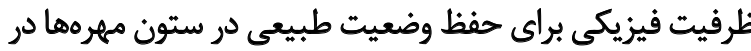

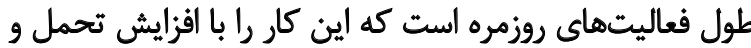

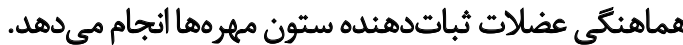
به همين خاطر تمرينات قدرتى و استقامت ناحيه مركزى بين

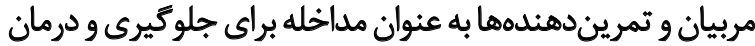

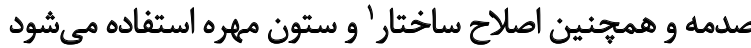

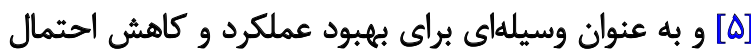

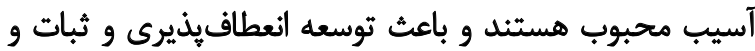

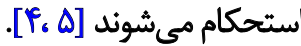

همجنين توانايى افراد در حفظ تعادل، تقريباً براى انجام

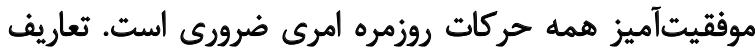

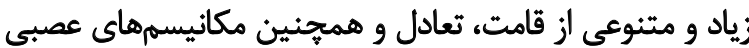

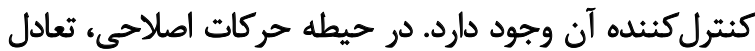

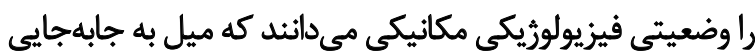
مركز ثقل در محدوده سطح اتكا در حد مطلوب رابي را دارد. بر اساس نظريه سيستمها، توانايى كنترل قامت در فضا ناشيى

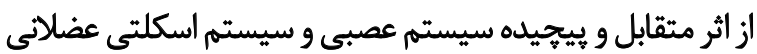

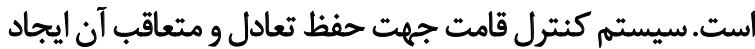

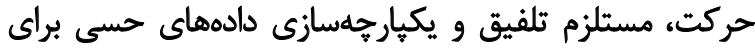

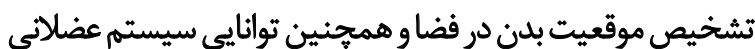

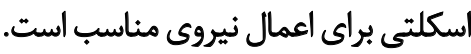
اجزاى سيستم عضلانى اسكلتى شامل دامنه حركتى،

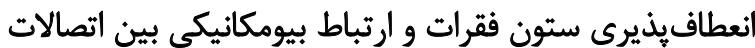

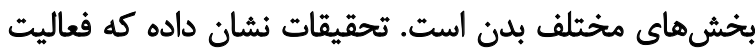

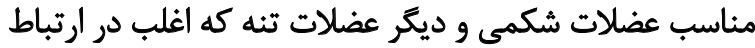

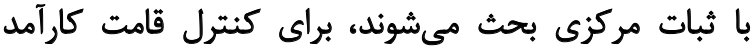
ضرورى است [؟]. از طرفى ارزيابى حركت، به متخصصان سلامت و آمادگى 
او ثبت شد. از اين آزمون به منظور ارزيابى انعطافيذيرى كلى

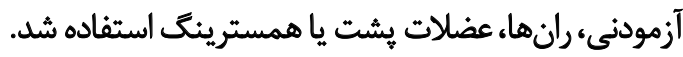

جهت اجراى آزمون دوى بيست متر سرعت، با علامت كثذارى

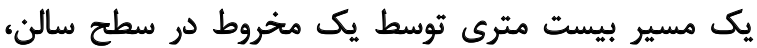

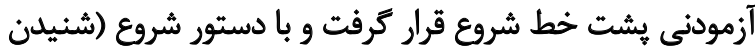

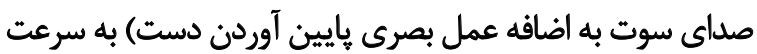

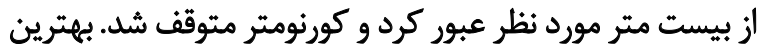

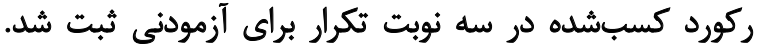

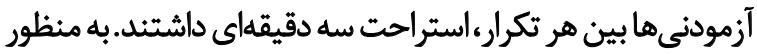
اندازميرى سرعت فرد در دوى سرعت از اين آزمون استفاده شد. روش اجراى آزمون برش عمودى (سارجنت) به اين شكل بود

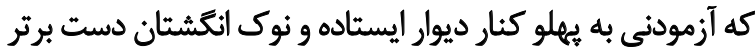

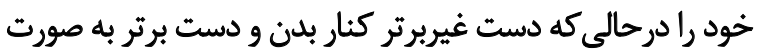

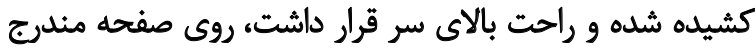

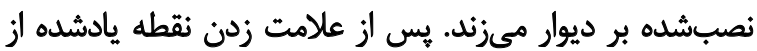

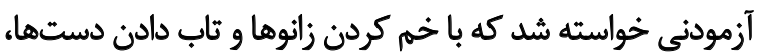

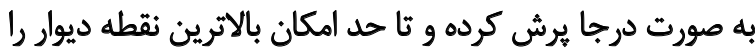
در اوج برش علامت بزند.

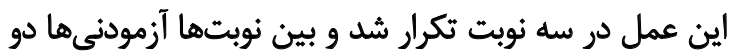

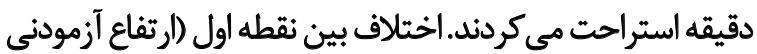

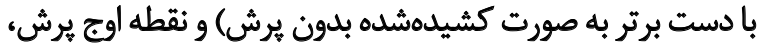

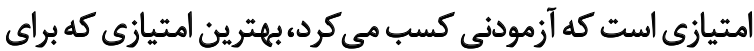

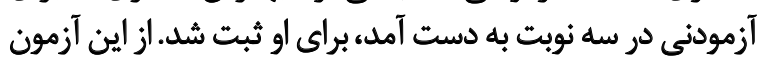
به منظور اندازهكيرى توان عضلات بـا استفاده شد. براي انجام آزمون تى اصلاحشده، ابتدا جههار مخروط طورى در در

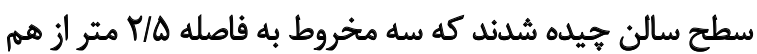

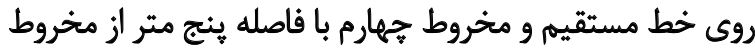
ميانى قرار ترفتند تا تشكيل حرف تي را بدهند.

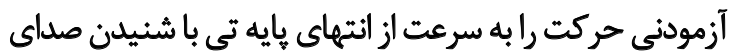

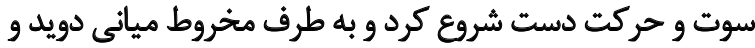

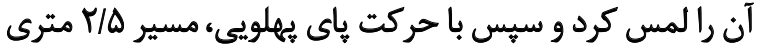

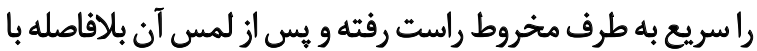

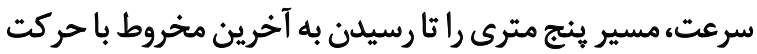

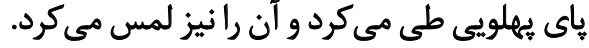

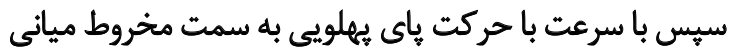

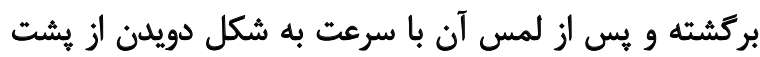

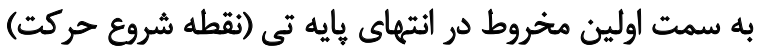

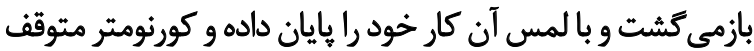

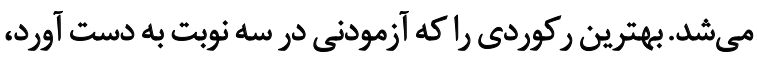

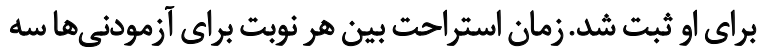

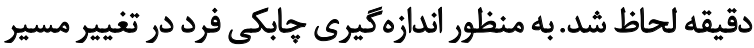
با سرعت از اين آزمون استفاده شد.
مانع، تشك و تخته (جهت ارزيابى آزمونهاى غربالكرى حركتى)،

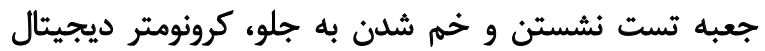

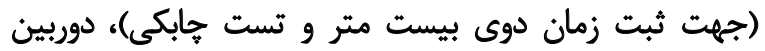

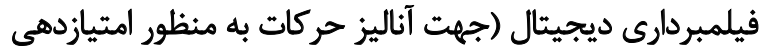

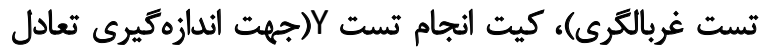
يويا) مورد نياز بود. روش اجرا در ابتدا بر اساس معيارهاي ورود به تحقيق از بين داوطلبانى

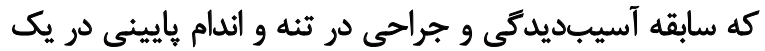

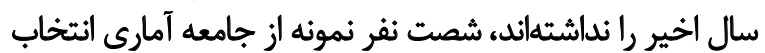

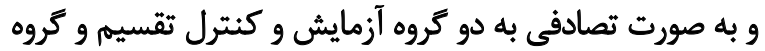

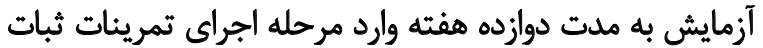

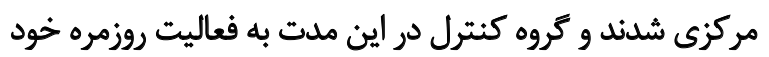

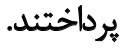

معيارهاي ورود به اين يُروهش شامل نداشتن سابقه آسيب در

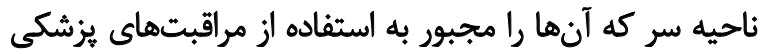

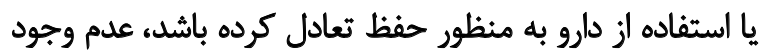

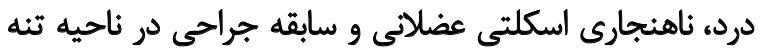

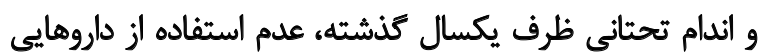

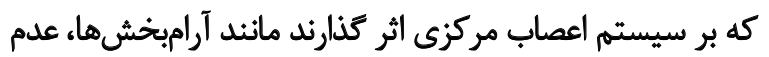

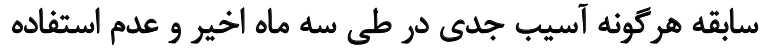

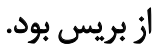

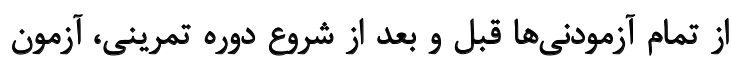

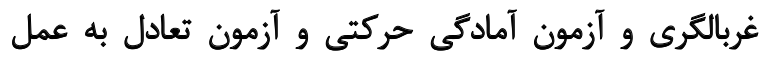

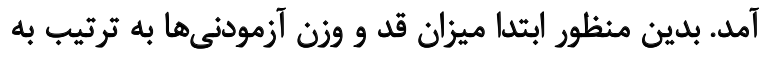
سانتىمتر و كيلوكرم اندازهيرى شدئ

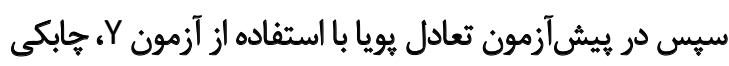

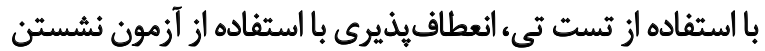

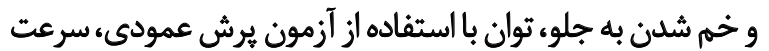

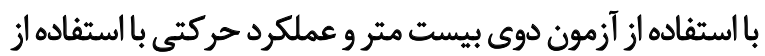

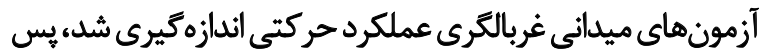

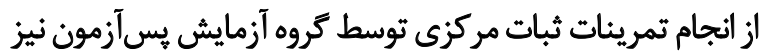

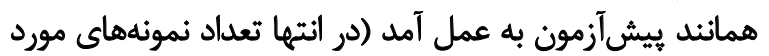

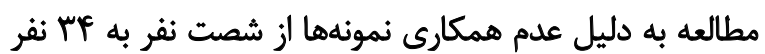

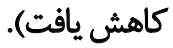

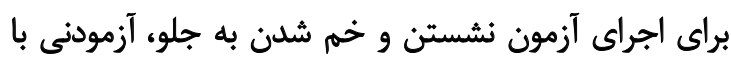

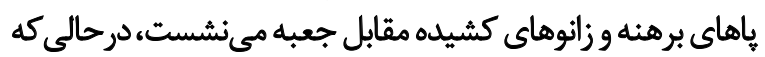

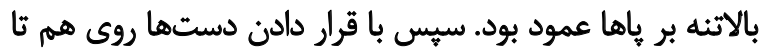

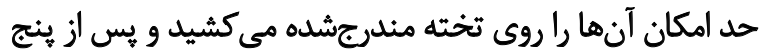

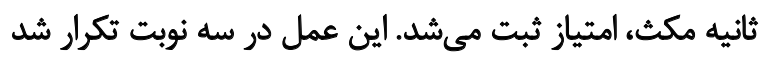

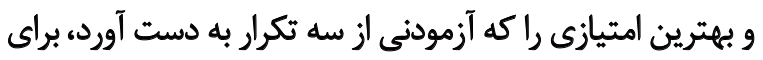




\section{The Functional Movement Screen}

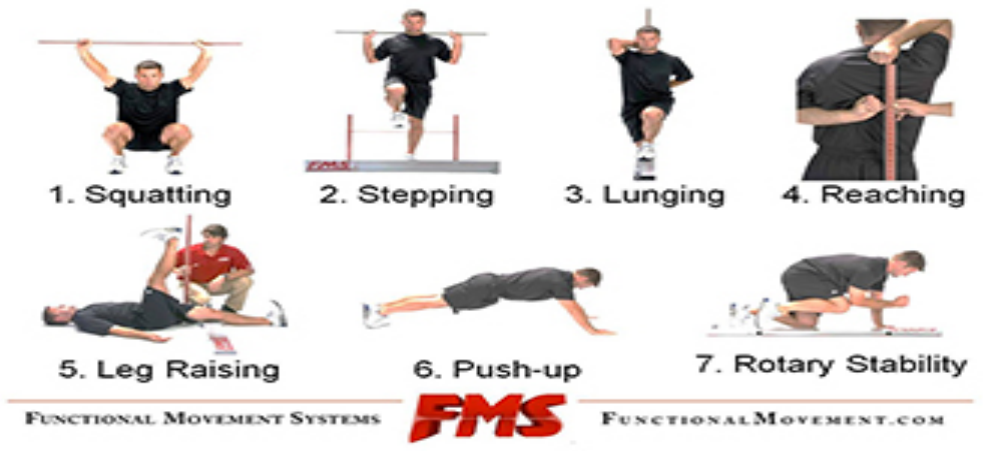

مجله بيومكانيك وزنث

تصوير آ. آزمونهاى عملكرد حركتى

شامل آزمونهاي ديب اسكات، كام برداشتن از روى مانع، لانج،

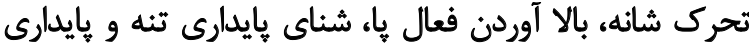

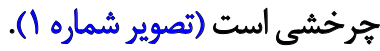

بس از هر حركت، يك امتياز بر اساس معيارهاي خاص

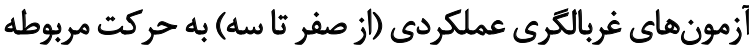

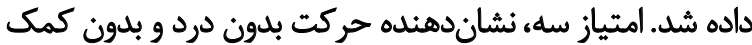

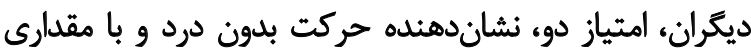

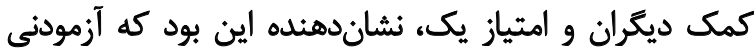

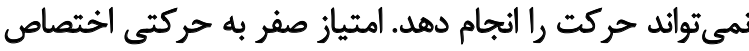
داده مي شد كه حامل درد بود.

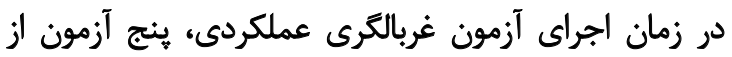

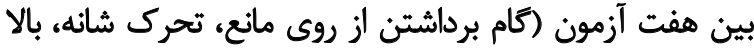

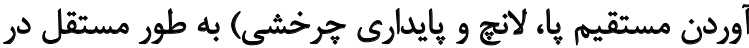

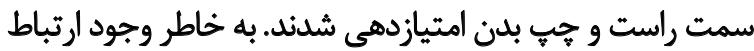

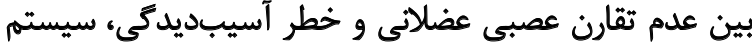

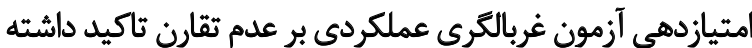

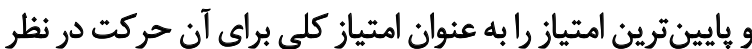

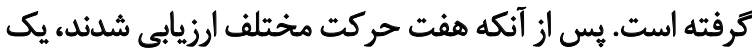

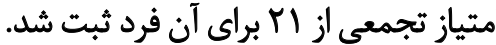

$$
\text { يروتكل تمريناث }
$$

تمرينات ثبات مركزى به طور مستمر به مدت دوازده هفته،

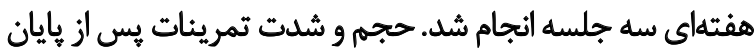

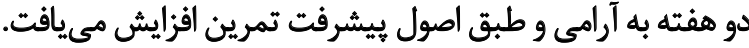
طول هر جلسه تمرين جهل دقيق آلهول بوديث. در هر جلسه ابتدا آزمودنىها با راه رفتن سريع و تمرينات

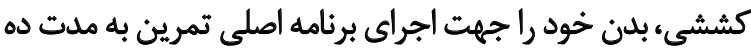

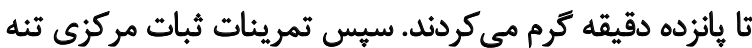

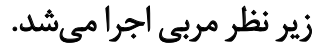

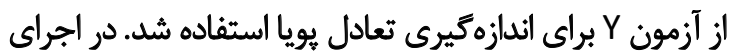

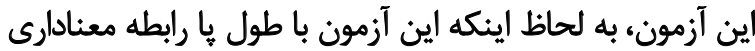

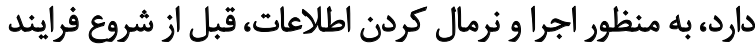
اندازه

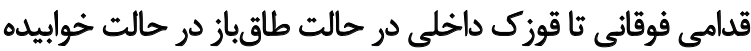

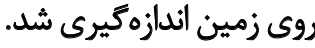

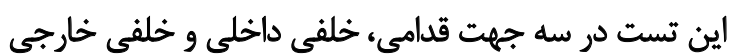

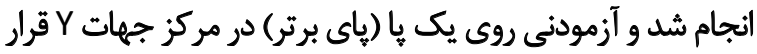

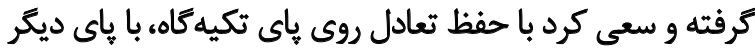

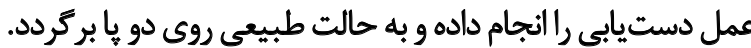

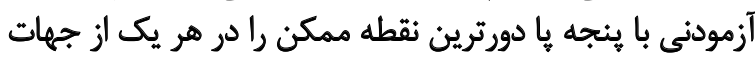

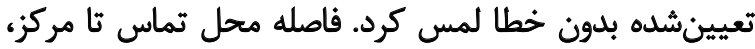
فاصله دستيابى بود كه به سانتىمتر اندازمكيرى شدر. فاصله محل تماس

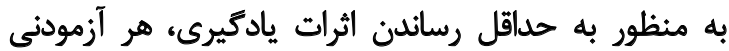

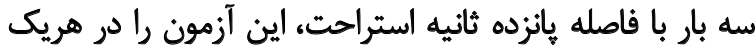

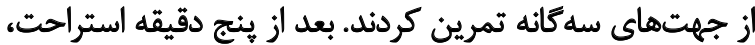

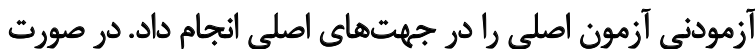

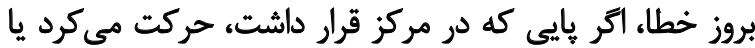

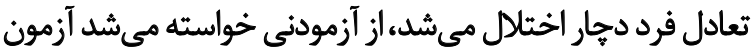

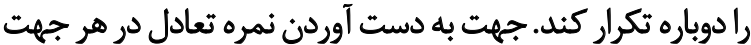

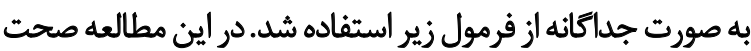

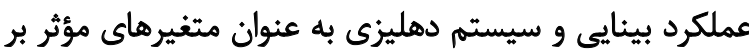
تعادل بررسى نشد (فرهول شماره ()).

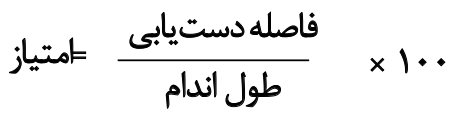

جهت انجام آزمونهاي عملكرد حركتى، هر آزمودنى بر اساس

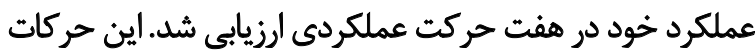


جدول ا. مشخصات جمعيتشناختى آزمودنىها (انحراف استانداردثميانكين)

\begin{tabular}{|c|c|c|c|c|}
\hline \multicolumn{4}{|c|}{ ميانيخن+|نحرافمعيار } & \multirow{2}{*}{ كَروهموردمطالعه } \\
\hline وزن (كيلوَّرم) & قد(سانتىمتر) & سن (سال) & تعداد & \\
\hline$\varepsilon N \cdot \wedge \pm V / 11$ & $|\varepsilon \cdot| \Lambda r \pm g / T \mid$ & $r \Delta / q T \pm T / \cdot r$ & 11 & آزمايشى (تمرينات ثبات مركزى) \\
\hline$V \backslash / V V \pm \Delta / r q$ & $1 \Delta 9 / \Delta \Lambda \pm F / q r$ & $r / / A r \pm T / \cdot r$ & 19 & كنترل \\
\hline
\end{tabular}

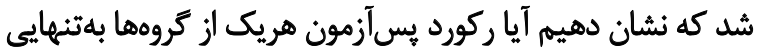

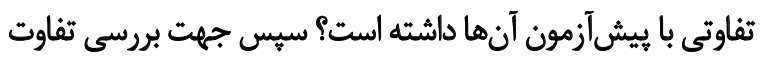

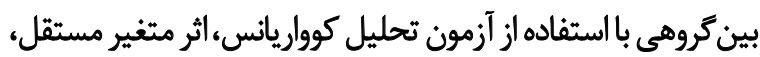

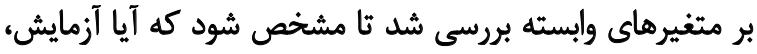

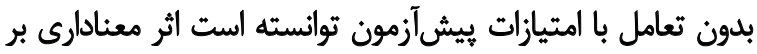

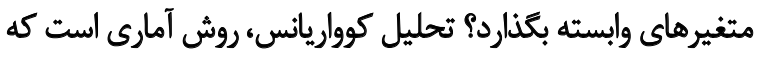

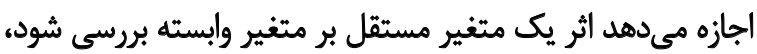

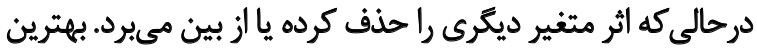

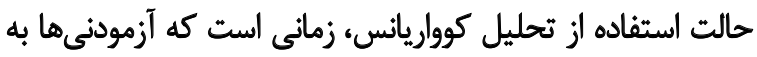

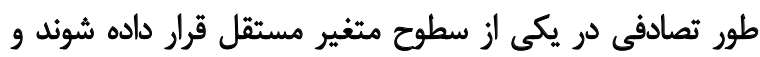

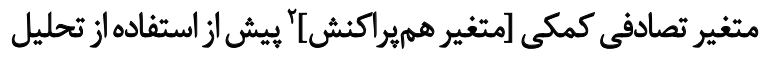

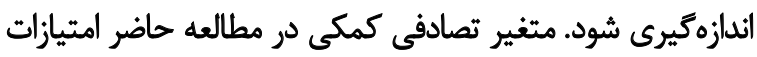

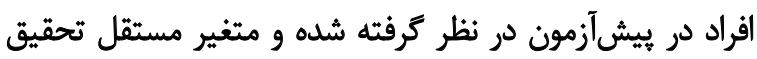

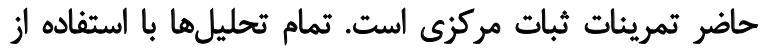

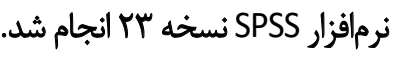

2. Covariate
اين غيروتكل تمرينى بر اساس تمرينـات ثبات مركزى

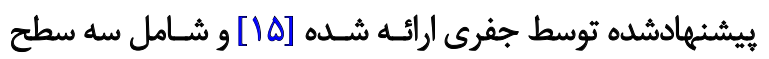

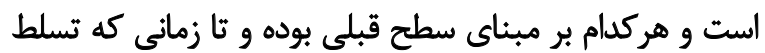

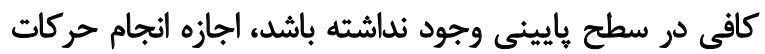

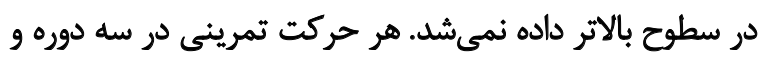

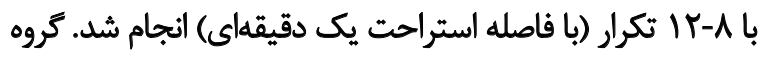

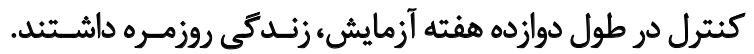

$$
\text { روش آمارى }
$$

از آمار توصيفى (از قبيل ميانكين و انحراف استاندارد) براى

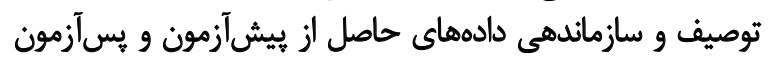

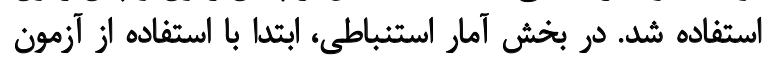

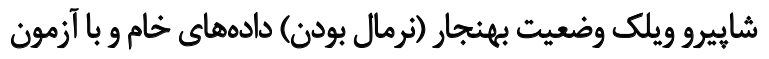

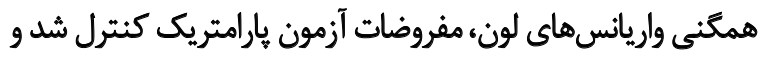

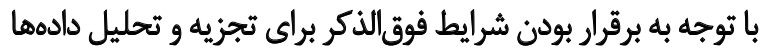

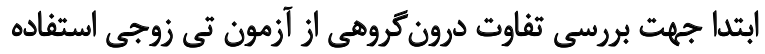

جدول r. نتايج آزمون تيزوجى درون كروهى در مراحل بيش آزمون و يسآزمون

\begin{tabular}{|c|c|c|c|c|c|c|}
\hline \multirow{2}{*}{ سطح معنادارى } & \multirow{2}{*}{ ارزش تى } & \multirow{2}{*}{ اختلاف امثياز درون تروهى } & \multicolumn{2}{|c|}{ ميانكين+انحراف معيار } & \multirow{2}{*}{ توروه } & \multirow{2}{*}{ مثغير } \\
\hline & & & يس آزمون & ي ييش آزمون & & \\
\hline.$/ 19$ & 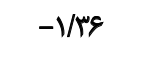 & $-\cdot / \Delta \cdot \pm 1 / 48$ & $\mid r / q \Psi \pm . / q T$ & $\mid r / \Psi r \pm . / \Delta F$ & كتترل & \multirow{2}{*}{$\begin{array}{c}\text { عملكردحركتى } \\
\text { (FMS) }\end{array}$} \\
\hline$\%+1$ & IV/AF & $\varphi / \Delta \cdot \pm V / \& \Delta$ & $\mid V / r T \pm+/ F \Delta$ & $\mid r / M \pm+/ \mathbb{Q} \Lambda$ & 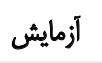 & \\
\hline.$/ \mu f$ &.$- / \mathrm{M}$ & $-. / \Delta \Delta \pm \Gamma / \Lambda$. & $V \in / T \Delta \pm T / \widetilde{R}$ & $V \Delta / \rho q \pm r / \Delta$. & 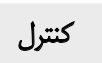 & \multirow{2}{*}{ ت تعادل } \\
\hline $.1 . .1$ & $-8 / \pi$ & $r / r q \pm T / q$ & $A \cdot / A H \pm Y+Y$ & $V \varepsilon / P A \pm T / R T$ & 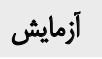 & \\
\hline.$\mu$ &.$- / N$ & $-. /|K| \pm . / \Delta r$ & $11 / \%+ \pm . / p q$ & $11 / r \pm \cdot / P A$ & 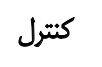 & \multirow{2}{*}{ سرعت } \\
\hline $.1 . .1$ & V/AV & $V / r \Delta \pm \cdot M r$ & $N \cdot \Psi \pm \cdot / V$ & $9 / 4 \cdot \pm \cdot / r 8$ & آزمايش - & \\
\hline.$/ \%$ &.$- / W$ & $-. / r \Delta \pm V / T q$ & $r \cdot / \cdot . \pm / v \Delta$ & $19 / V \Delta \pm . / 98$ & كتترل & \multirow{2}{*}{ ت توان } \\
\hline $.1 . .1$ & - NaY & $-r / / \& \pm V / \Delta$. & $r r / q F \pm . / V E$ & $19 / \mathrm{W} \pm \cdot / \Delta \mathrm{V}$ & آزمايش & \\
\hline .119 & $1 / r v$ & $-\cdot / M \pm \pm \cdot / \Delta r$ & $\mid \varphi / \uparrow \Psi+/ \varphi \varphi$ & $\mid \varphi / T Y \pm . / \varphi$ & كتترل & \multirow{2}{*}{ جابكى } \\
\hline $.1+.1$ & r/ar & $V / \wedge \Delta \pm V / e q$ & $11 / v a \pm 1 / * r$ & $\mid r / g x \pm \cdot / T T$ & 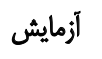 & \\
\hline.$/ 4 F$ &.$/ 97$ & $. / F Y \pm 1 / \mathrm{VA}$ & $r / A Y \pm \cdot / A r$ & $r r / r \mid \pm . / N$ & كتترل & \multirow{2}{*}{ انعطافيذيرى } \\
\hline.$\% 1$ & -91.4 & $-r / . \pm \pm r / 11$ & TNFI士./VA & YQ/\&।I./9Y & آزمايش آ & \\
\hline
\end{tabular}

مجلله بيومكانيك ورنث 
جدول rا. نتايج آزمون خطى بودن همبستكى متغير هميراكنش و مستقل

\begin{tabular}{|c|c|c|c|c|}
\hline $\mathbf{P}$ & $\mathbf{F}$ & درجه آزادى & مجموع مجذورات & كروه \\
\hline $.1 .+1$ & $F \Delta / M$ & 1 & $95 / 94$ & ييش آزمون عملكرد حركتى \\
\hline $.1 .+1$ & TINT & 1 & rVT/Ar & ييش آزمون تعادل \\
\hline $.1 .+1$ & $\mid N \Delta / \% \Delta$ & 1 & $V \cdot / A T$ & ييش آزمون سرعت \\
\hline $.1 . .1$ & $15+/ 4$ & 1 & $r \Delta F / F \Delta$ & ييش آزمون توان \\
\hline $.1 . .1$ & $\pi / 19$ & 1 & EN.FE & ييش آزمون جابكى \\
\hline $.1++1$ & $V \Delta / 11$ & 1 & rra/rr & ييش آزمون انعطافيذيرى \\
\hline
\end{tabular}

بهدستآمده، حاكى از آن است كه بيشفرض همكنى واريانسها

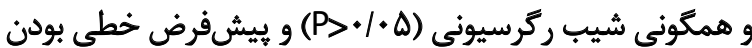

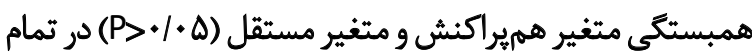
متغيرهاى يرُوهش نيز برقرار است.

در بررسى نتايج آزمون بيش فرض خطى بودن همبستكى متغير

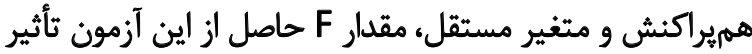

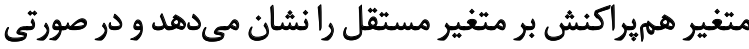

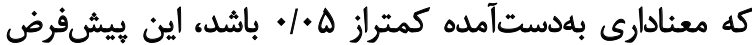

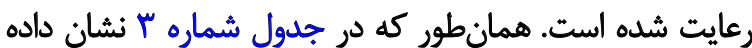

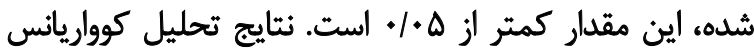

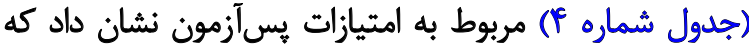

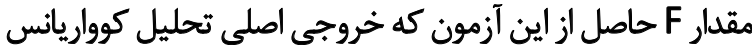

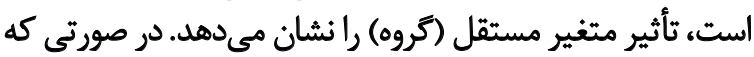

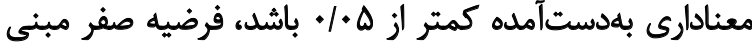

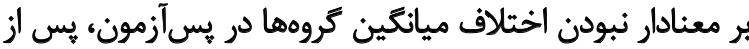

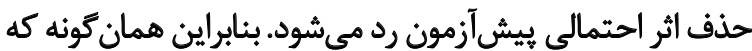

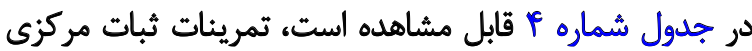

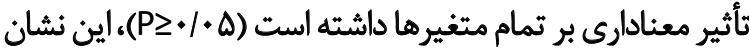

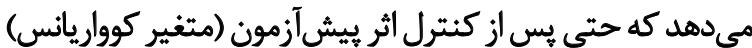

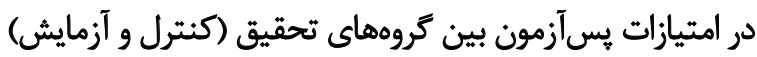

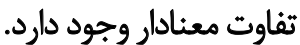

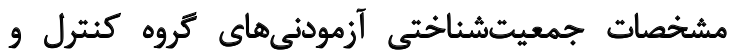

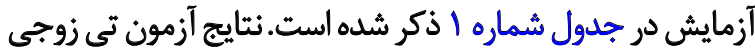

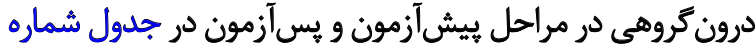

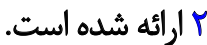

نتايج آزمون تى نشان ميىهد كه متعاقب دوازده هفته تمرين

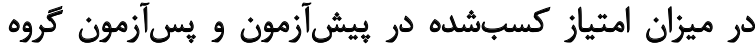

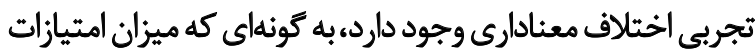

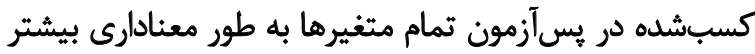

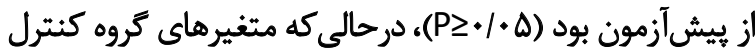

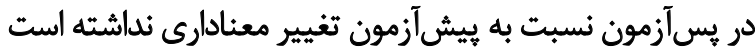

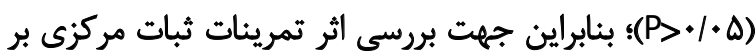

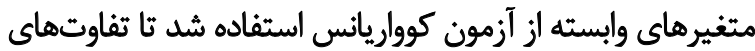

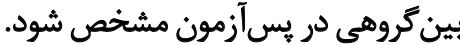
اجراى تحليل كوواريانس در مطالعه حاضر مستلزم اين است مثرئ

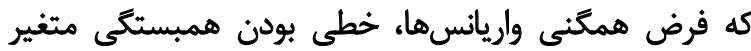

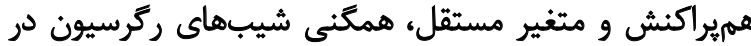
متغيرهاي تحت بررسى رعايت شده باشدي. در ادامه به بررسى اين بيشفرضها يرداخته شد و نتايج

جدول f. تحليل كوواريانس يكراهه جهت بررسى اثربخشى تمرينات ثبات مركزى بر امتيازات متغيرهاى وابسته

\begin{tabular}{|c|c|c|c|c|c|}
\hline قدرت مشاهدهشده & $\mathbf{P}$ & $\mathbf{F}$ & درجه آزادى & مجموع مجذورات & كروه \\
\hline.$/ 199$ & $.1 . .1$ & EgIVA & 1 & $|f \cdot| M \mid$ & عملكرد حركتي \\
\hline.$/ 91$ & $.1 . .1$ & Ifepter & 1 & ITH/V. & ت تعادل \\
\hline.$/ 94$ & $.1 . .1$ & $\Delta 1 / W$ & 1 & $19 / 20$ & سرعت \\
\hline - /AF & $.1 . .1$ & MelAF & 1 & $\mathrm{~W} / \mathrm{Aq}$ & 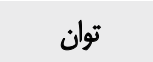 \\
\hline.$/ 8 V$ & $.1 . .1$ & $18 / 1$ & 1 & m/va & جابكى \\
\hline - Af &.$/ . r$ & rNFA & 1 & ITr/a. & اثعطاف هيذيرى \\
\hline
\end{tabular}

مجله بيومكانيك ورنشي 
همجينين نتايج حاصل با نتايج يُروهشهاي اماى و همكاران،

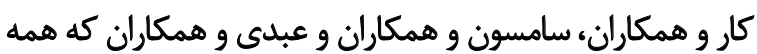

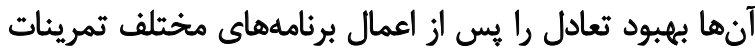

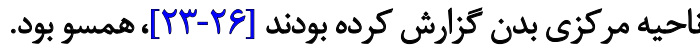
از طرفي، نتايج تحقيق حاضر با نتايج بركرفته از تحقيق

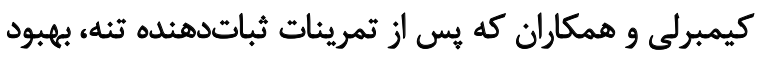

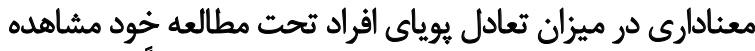

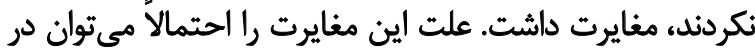

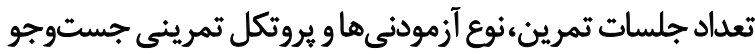

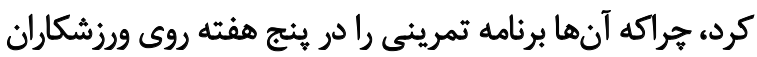
تنيس اجراكردماند.

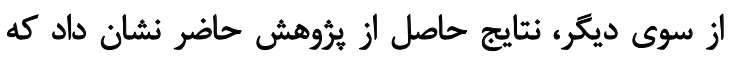

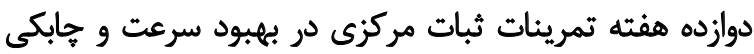

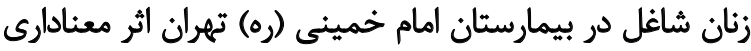

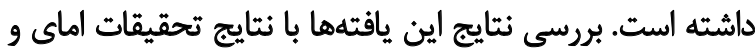

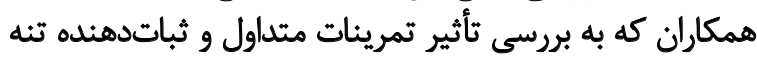

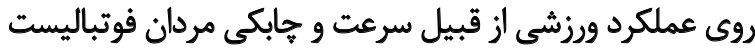

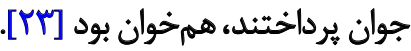

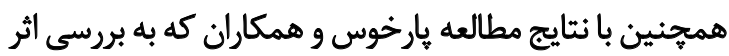

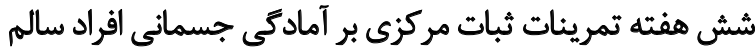

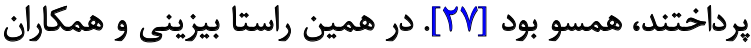

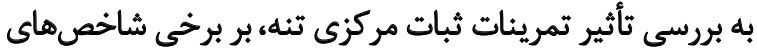

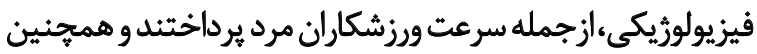

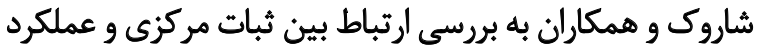

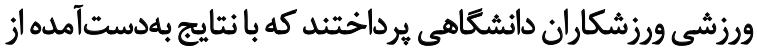

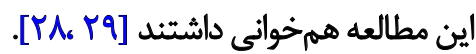

شيلينك و همكاران، تأثيرات تمرينات قدرتى و استقامتى ثبات

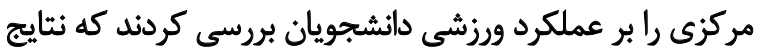

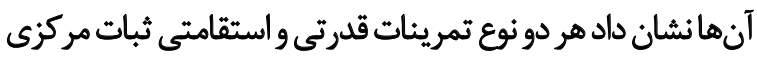

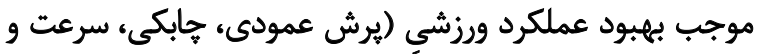

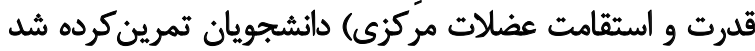

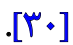

همجينين مايلز و همكاران طى مطالعهاى با عنوان بررسى اثرات

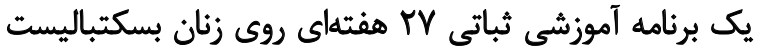

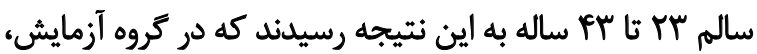

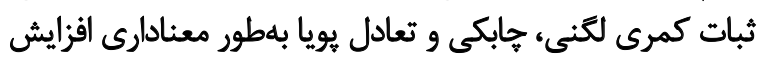

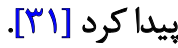

تمرينات ثباتى، در بهبود قدرت كانسنتريك عضله عرضى

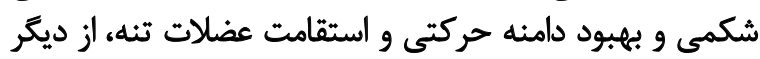

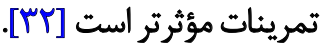

اين تمرينات باتأييد در برانكيختكى عضلات سكمنتال (عضلات
هدف از مطالعه حاضر، بررسى تأثير تمرينات ثبات مركزى بر

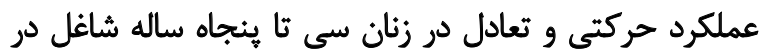

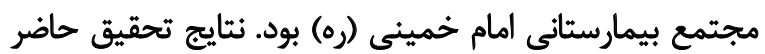

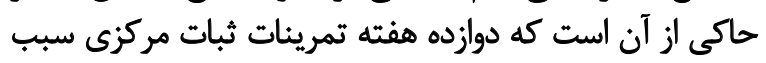

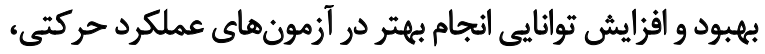

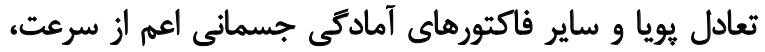

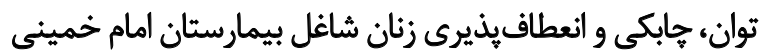

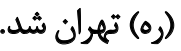

در ارتباط با تأثير تمرينات ثبات مركزى بر بهببود عملكرد بردي

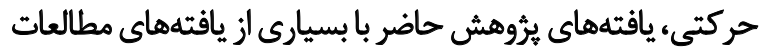

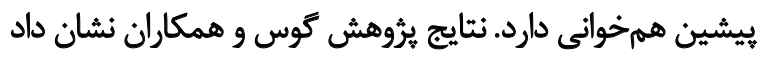

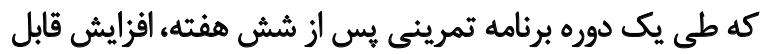

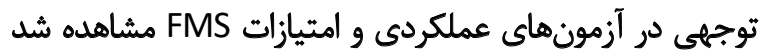

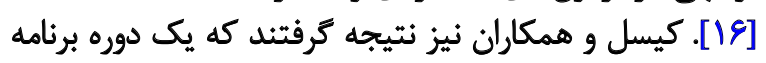

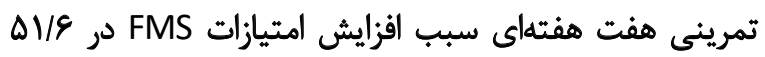

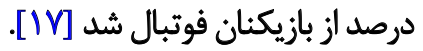

از طرفى شرى و همكاران طى مطالعهاى اعلام كردند كه فعال

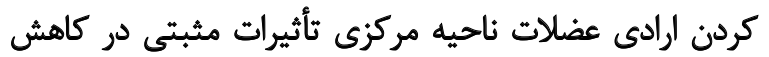

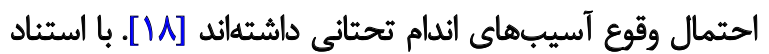

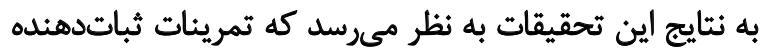

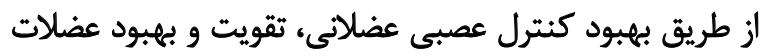

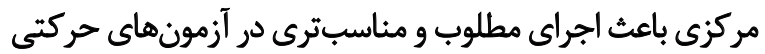

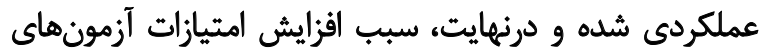
حركتى عملكردى مى شودود.

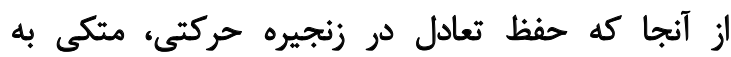

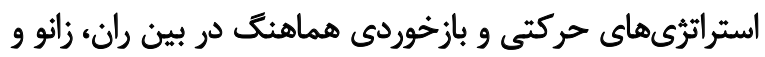

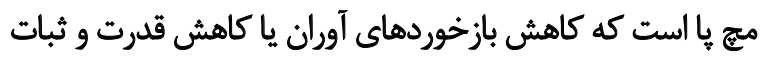

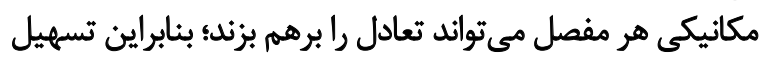

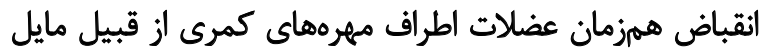

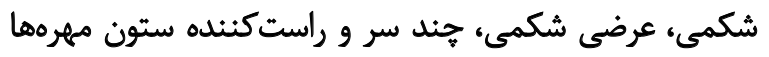

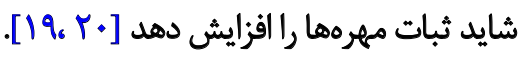

در همين راستا، طى مطالعه حاضر با بهكاركيرى تمرينات

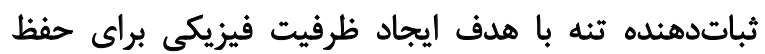

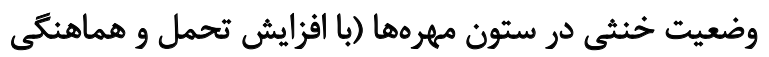

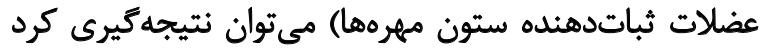

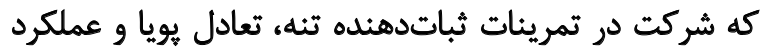
فرد را بهبود مي شيخشد. نتيجه حاصل با نتايج تحقيقات جانسون و همكاران و كاريس و و

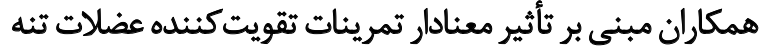

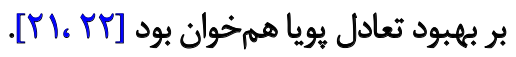


مركزى تنه مي شوند، درنتيجه كسب نتايج بهتر در انعطافيذيرى

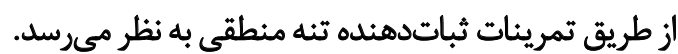

\section{تتيجهيَيرىنهايى}

با توجه به نتايج حاصل از يروهش حاضر مى توان نتيجه كرفت

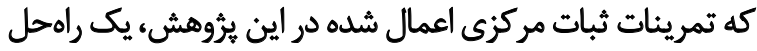

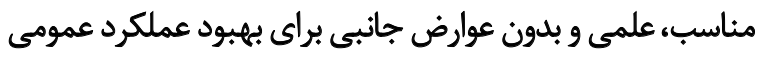

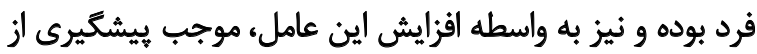
آسيبديدكى فرد مىشوند.

در واقع، تمرينات ثباتدهنده كارايى سيستم عصبى عضلانى را

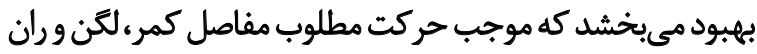

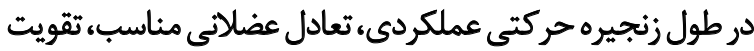

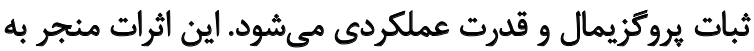

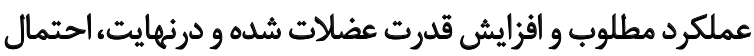
وقوع آسيب را كاهش مي دهند.

ملاحظات اخلاقى

\section{ي اييروى از اصول اخلاق يثوهش}

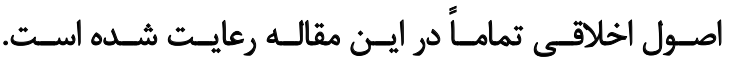

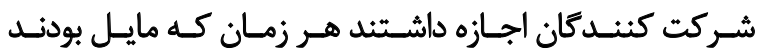

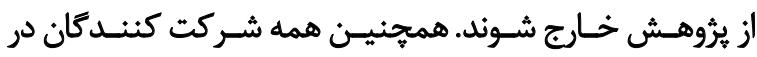

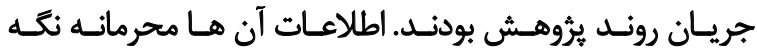

$$
\text { داشـته شــد. }
$$

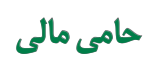

اين مقاله بركرفته از طرح بروهشى اجرايى در معاونت يثروهشى

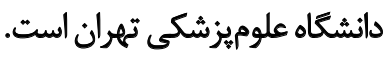

$$
\text { مشاركت نويسندكًان }
$$

تمام نويسندكان در طراحى، اجرا و نكارش همه بخشهاى يُروهش حاضر مشاركت داشتهاند.

$$
\text { تعارض مناقع }
$$

بنابر اظهار نويسندكان اين مقاله تعارض منافع ندارد.

$$
\text { تشكر و قدردانى }
$$

از زحمات بىشائبه همه بزركوارانى كه در راستاى اجراى

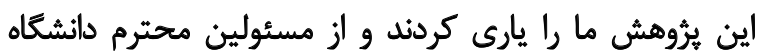

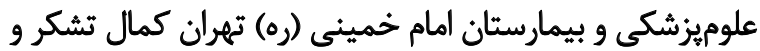
قدردانى را داريم.
ريز درون مفاصل ستون فقرات)، منجر به افزايش ثبات سكمنتال

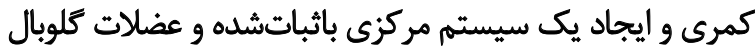

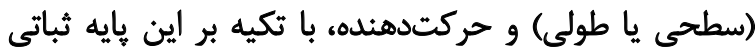

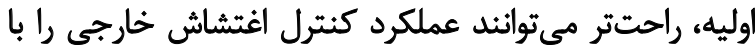

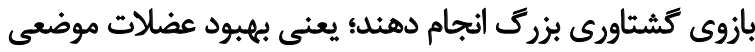

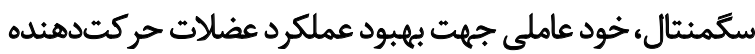

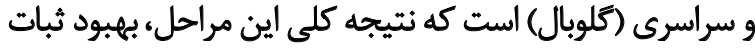

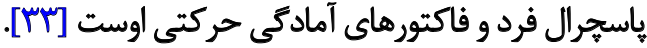
نتايج بهدستآمده نشان داد كه انجام عب جلسهاي تمرينات

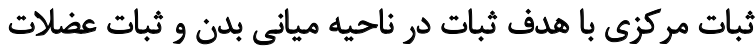

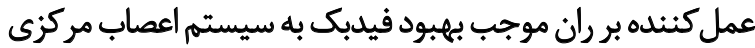

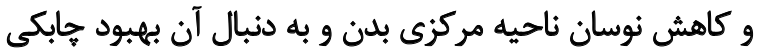

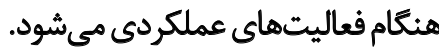

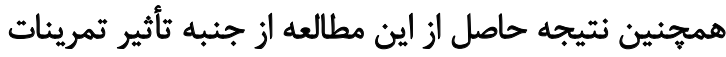

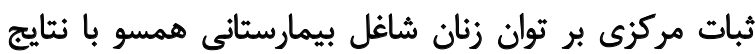

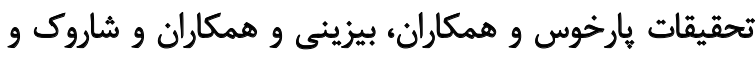

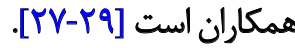

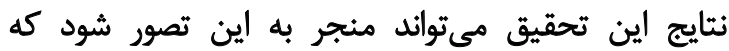

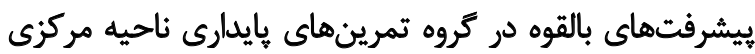

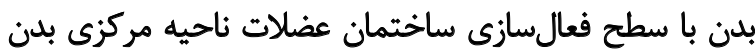

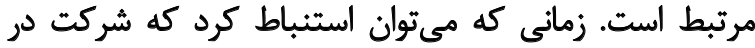

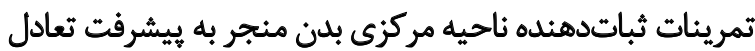

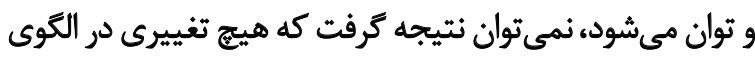
فعال سازى عضلات تجربه نمى تشود.

هرجند كه به تحقيقات بيشترى در مورد اين تمرينات احساس

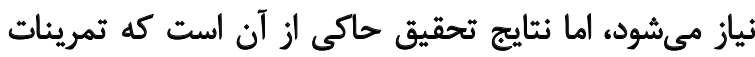

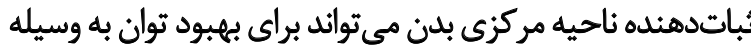

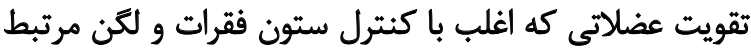

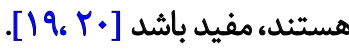

متغير ديكرى كه در اين يرّوهش ارزيابى شد، انعطافيذيرى

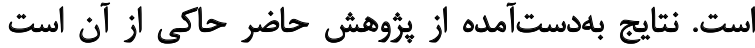

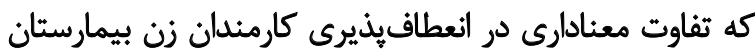

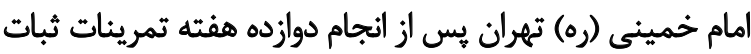

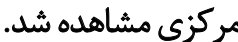
نتيجه حاصل بانتيجه تحقيق شيلينگ و همكاران كه تأثيرات

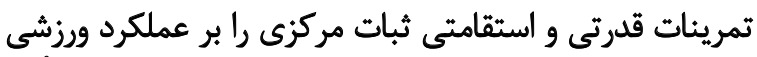

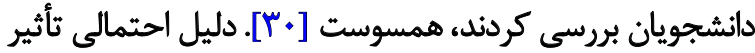

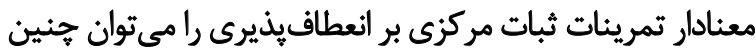

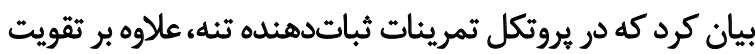

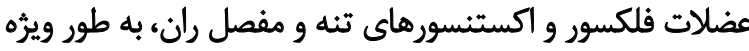

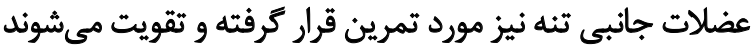
و از اين طريق منجر به افزايش قدرت و و عملكرد مطلوب در نقريت ناحيه 


\section{Refrences}

[1] Ali Arabian F, Motamedzade M, Golmohammadi R, Moghim Beigi A, Pir Hayati F. [The impact of ergonomics intervention on musculoskeletal disorders among Nahavand Alimoradian hospital staff (Persian)]. Iran J Ergon. 2013; 1(1):23-32. http://journal.iehfs.ir/article-1-23-en.html

[2] Heyward VH. Advanced fitness assessment and exercise prescription. $6^{\text {th }}$ ed. Champaign: Human Kinetics; 2010. https://books.google.com/ books?id=5BheygAACAAJ \&dq

[3] Granacher U, Schellbach J, Klein K, Prieske O, Baeyens JP, Muehlbauer $\mathrm{T}$. Effects of core strength training using stable versus unstable surfaces on physical fitness in adolescents: A randomized controlled trial. BMC Sports Sci Med Rehabil. 2014; 6:40. [DOI:10.1186/2052-1847-6-40] [PMID] [PMCID]

[4] Nesser TW, Huxel KC, Tincher JL, Okada T. The relationship between core stability and performance in division I football players. J Strength Cond Res. 2008; 22(6):1750-4. [DOI:10.1519/JSC.0b013e3181874564] [PMID]

[5] Kang KY. Effects of core muscle stability training on the weight distribution and stability of the elderly. J Phys Ther Sci. 2015; 27(10):3163-5. [DOI:10.1589/jpts.27.3163] [PMID] [PMCID]

[6] Freeman JA, Gear M, Pauli A, Cowan P, Finnigan C, Hunter H, et al. The ef fect of core stability training on balance and mobility in ambulant individuals with multiple sclerosis: A multi-centre series of single case studies. Mult Scler. 2010; 16(11):1377-84. [DOI:10.1177/1352458510378126] [PMID]

[7] McGill SM, Andersen JT, Horne AD. Predicting performance and injury resilience from movment quality and fitness scores in a basketball team over 2 years. J Strength Cond Res. 2012; 26(7):1731-9. [DOI:10.1519/ JSC.0b013e3182576a76] [PMID]

[8] Cook G, Burton L, Hoogenboom B. Pre-participation screening: The use of fundamental movements as an assessment of function - part 1. N Am J Sports Phys Ther. 2006; 1(2):62-72. [PMID] [PMCID]

[9] Song HS, Woo SS, So WY, Kim KJ, Lee J, Kim JY. Effects of 16-week functional movement screen training program on strength and flexibility of elite high school baseball player. J Exerc Rehabil. 2014; 10(2):124-30. [DOI:10.12965/jer.140101] [PMID] [PMCID]

[10] Peate WF, Bates G, Lunda K, Francis S, Bellamy K. Core strength: A new model for injury prediction and prevention. J Occup Med Toxicol. 2007; 2:3. [DOI:10.1186/1745-6673-2-3] [PMID] [PMCID]

[11] Ghasempoor Kh, Rahnama N, Bagherian-Dehkordi S. [Functional movment screening of students of Shahrekord technical Institute, Iran, for sports injuries prevention (Persian)]. J Res Rehabil Sci. 2015; 11(4):26372. http://jrrs.mui.ac.ir/index.php/jrrs/article/view/2391

[12] Perry FT, Koehle MS. Normative data for the functional movement screen in middle-aged adults. J Strength Cond Res. 2013; 27(2):458-62. [DOI:10.1519/JSC.0b013e3182576fa6] [PMID]

[13] Khoramian M, Golmohammadi B, Akbari H. [The effect of the selective balanced on ball and core resistance training program on the older females (Persian)]. Mot Behav. 2015; 7(19):105-22. https://mbj.ssrc.ac.ir/ article_343.html

[14] Chorba RS, Chorba DJ, Bouillon LE, Overmyer CA, Landis JA. Use of a functional movement screening tool to determine injury risk in female collegiate athletes. N Am J Sports Phys Ther. 2010; 5(2):47-54. [PMID] [PMCID]

[15] Jeffreys I. Developing a progressive core stability program. Strength Cond J. 2002; 24(5):65-6. [DOI:10.1519/00126548-200210000-00017]
[16] Goss DL, Christopher GE, Faulk RT, Moore J. Functional training program bridges rehabilitation and return to duty. J Spec Oper Med. 2009; 9(2):29-48. [PMID]

[17] Kiesel K, Plisky P, Butler R. Functional movement test scores improvefollowing a standardized off-season intervention program in professional football players. Scand J Med Sci Sports. 2011; 21(2):287-92.[DOI:10.1111/j.1600-0838.2009.01038.x] [PMID]

[18] Shirey M, Hurlbutt M, Johansen N, King GW, Wilkinson SG, Hoover $D L$. The influence of core musculature engagement on hip and knee kinematics in women during a single leg squat. Int J Sports Phys Ther. 2012; 7(1):1-12. [PMID] [PMCID]

[19] Sahebozamani M, Mohammad Ali Nasab E, Daneshmandi H. [Effect of core stability training on the trunk endurance of indoor soccer players (Persian)]. Stud Sport Med. 2014; 6(15):15-28. https:// smj.ssrc.ac.ir/article_35.html

[20] Hägglund $M$, Waldén $M$, Ekstrand J. Previous injury as a risk factor for injury in elite football: A prospective study over two consecutive seasons. Br J Sports Med. 2006; 40(9):767-72. [DOI:10.1136/ bjsm.2006.026609] [PMID] [PMCID]

[21] Johnson EG, Larsen A, Ozawa H, Wilson CA, Kennedy KL. The ef fects of Pilates-based exercise on dynamic balance in healthy adults. J Bodyw Mov Ther. 2007; 11(3):238-42. [DOI:10.1016/j. jbmt.2006.08.008]

[22] Carpes FP, Reinehr FB, Mota CB. Effects of a program for trunk strength and stability on pain, low back and pelvis kinematics, and body balance: A pilot study. J Bodyw Mov Ther. 2008; 12(1):22-30. [DOI:10.1016/j.jbmt.2007.05.001] [PMID]

[23] Imai A, Kaneoka K, Okubo Y, Shiraki H. Effects of two types of trunk exercises on balance and athletic performance in youth soccer players. Int J Sports Phys Ther. 2014; 9(1):47-57. [PMID] [PMCID]

[24] Carr JH, Shepherd RB. Stroke rehabilitation: Guidelines for exercise and training to optimize motor skill. Oxford: Butterworth-Heinemann; 2003. https://books.google.com/ books?id=AccPvgAACAAJ\&dq

[25] Samson KM. The effects of a five-week core stabilization-training program on dynamic balance in tennis athletes [MSc. thesis]. Morgantown, WV: West Virginia University; 2005. https://www.proquest.com/openview/6566ad2d8d89fe10c0797b81963e4f87/1

[26] Abdi J, Sadeghi H. The effect of eight-week core stability training program on the dynamic balance in young elite footballers. Scolio sis. 2013; 8:P20. [DOI:10.1186/1748-7161-8-S1-P20] [PMCID]

[27] Parkhouse KL, Ball N. Influence of dynamic versus static core exercises on performance in field based fitness tests. J Bodyw Mov Ther. 2011; 15(4):517-24. [DOI:10.1016/j.jbmt.2010.12.001] [PMID]

[28] Bizzini M, Impellizzeri FM, Dvorak J, Bortolan L, Schena F, Modena $R$, et al. Physiological and performance responses to the "FIFA $11+"$ (part 1): Is it an appropriate warm-up? J Sports Sci. 2013; 31(13):1481-90. [DOI:10.1080/02640414.2013.802922] [PMID]

[29] Sharrock C, Cropper J, Mostad J, Johnson M, Malone T. A pilot study of core stability and athletic performance: Is there a relationship? Int J Sports Phys Ther. 2011; 6(2):63-74. [PMID] [PMCID]

[30] Schilling F, Murphy C, Bonney R, Thich L. Effect of core strength and endurance training on performance in college students: Randomized pilot study. J Bodyw Mov Ther. 2013; 17(3):278-90. [DOI:10.1016/j.jbmt.2012.08.008] [PMID] 
[31] Mills JD, Taunton JE, Mills WA. The effect of a 10-week training regimen on lumbo-pelvic stability and athletic performance in female athletes: A randomized-controlled trial. Phys Ther Sport. 2005; 6(2):60-6. [DOI:10.1016/j.ptsp.2005.02.006]

[32] Salavati M, Bagheri H, Ebrahimi Takamjani E, Mobini B. [Comparative study of biodex dynamic balance and limits of stability test in normal subject and patient with chranic lowback pain (Persian)]. Razi J Med Sci. 2003; 9(32):699-708. http://rjms.iums.ac.ir/browse.php?a_ id $=1687 \&$ sid $=1 \&$ slc_lang $=e n$

[33] Ehsani F, Mohseni Bandpei MA, Shanbeh-Zadeh S. [The effect of stabilization exercises on objective outcome measures in patients with chronic non-specific low back pain: A systematic review with particular emphasis on randomized controlled clinical trial (Persian)]. Arch Rehabil. 2013; 14(2):8-21. http://rehabilitationj.uswr.ac.ir/article-1-1272-fa.html 
This Page Intentionally Left Blank 